\title{
LES ANGLICISMES FRANÇAIS RELATIFS AU CINÉMA : ENTRE LA TERMINOLOGIE, LES RECOMMENDATIONS OFFICIELLES ET L'USAGE
}

\author{
WERONIKA WOŹNIAK
}

\begin{abstract}
French anglicisms related to cinema: between terminology, official recommendations and usage

The complex terminology of cinematography, where French and foreign terms meet and compete, reflects the nature of this domain, which is the area of international cooperation, of the exchange of thoughts and expression. In order to avoid the compulsory usage of foreign nomenclature, the creation and distribution of French equivalents have become the assignment of institutions such as the Commission d'enrichissement de la langue française and l'Académie française. Despite the development of indigenous terminology in the field of cinema, which is strongly associated with the international film industry, especially the American one, the circulation of Anglicisms remains significant. The objective of this article is to study English terms in the aforementioned domain. Therefore, firstly, the official recommendations of the Commission d'enrichissement de la langue française and l'Académie française for the employment of French terms in the field of cinema are presented. The second part constitute the analysis of the presence of English terms from the corpus, such as casting, flashback, steadicam, trailer, in contemporary French dictionaries, in relation to the official recommendations of the aforementioned institutions. Finally, we focus on the presence of Anglicisms and their French equivalents in French journalistic discourse in the mass-circulation press, in order to study the circulation of loans words and to examine whether they are actually substituted by notions of French terminology.
\end{abstract}

Key words : Loan words, cinematography, terminology, journalistic discourse

Mot clés : Anglicismes, cinématographie, terminologie, discours journalistique 


\section{Introduction}

La cinématographie, le "septième art » selon l'expression forgée par Ricciotto Canu$\mathrm{do}^{1}$, comme plusieurs domaines artistiques et scientifiques, n'accepte ni frontières territoriales, ni culturelles ou linguistiques. Sans doute, constituant un champ de coopération internationale, nécessite-t-elle un langage commun d'échange d'idées. Malgré ses racines françaises dues aux frères Auguste et Louis Lumière, inventeurs du cinématographe et organisateurs de la première projection publique de film, qui sont considérés comme les premiers cinéastes dans l'histoire du cinéma, depuis longtemps la cinématographie dans la conscience collective est fortement associée à la culture américaine. En raison des influences culturelles et économiques de l'industrie cinématographique d'outre-Atlantique, d'où différentes techniques et tendances sont empruntées, c'est la langue anglaise qui a la plus grande incidence sur le "septième art ", car le besoin de dénommer ces phénomènes importés pousse les professionnels non anglophones à employer des termes d'origine anglo-américaine. Comme le remarque Jean Giraud dans son article «Terminologie du cinéma ", qui souligne que le domaine de la cinématographie se caractérise par un goût prononcé pour les emprunts, il y a longtemps que "le film américain commence à déferler sur nos écrans, avec une première vague de néologismes " (Giraud 1967 : 118). Ainsi, on aperçoit que la puissance technologique et l'imposition des tendances d'outre-Atlantique entraînent aussi la dominance langagière, car bien que la langue française ne soit pas complètement tributaire de l'anglais, la circulation des mots d'origine anglaise, utilisés par les cinéastes en réponse aux nouveaux phénomènes et problèmes, demeure significative. Les génériques des films français sont notamment à observer, car il y figure plusieurs termes concernant la production cinématographique qui ne possèdent pas d'équivalents autochtones, comme making of et voiture travelling.

Il est à noter cependant, que les génériques contiennent également des anglicismes qui peuvent être remplacés par des termes francophones officiellement recommandés, comme par exemple : casting director (au lieu de régisseur de distribution) ou steadicam (au lieu de stabilisateur). Le recours aux anglicismes n'est donc pas uniquement dû au manque du signifiant dans la langue d'adoption. Comme le remarque Geneviève Petiot, très souvent seules les fonctions principales sont traduites pendant que celles « de fin » ne le sont pas, ainsi la «traduction partielle » des génériques influence la propagation et l'emploi direct des termes étrangers (Petiot 1987 : 303). Qui plus est, il semble que dans ce domaine il soit aussi question de motivations socioculturelles, voire psychologiques, car d'un côté les anglicismes permettent aux cinéastes de s’adapter ainsi que de participer activement à un milieu multiculturel, et de l'autre côté ils véhiculent des connotations positives du rêve américain. Ainsi, soulignons avec Maurice Pergnier que dans le domaine de la cinématographie « (...) le désir d’américanisation qui s’y manifeste (que ce désir soit spontané ou artificiellement créé pour des raisons économiques) est une autre question.» (Pergnier 1989: 16).

Or, en France, le devoir constitutionnel de la protection de la langue française encourage les spécialistes, les linguistes et les professionnels de plusieurs branches, à la défense et à la promotion de la langue grâce à la création d'homologues français, entre autres,

Ricciotto Canudo, écrivain et critique franco-italien, utilise cette appellation dans le Manifeste $d u$ septième art, publié en 1923 dans La gazette des sept arts. 
dans le cadre de domaines spécialisés. Par conséquent, deux types de démarches sont entreprises : la prévention et la défense. Par le biais du décret n $72-19$ de 1972, les commissions qui ont pour but de « proposer des termes nécessaires soit pour désigner une réalité nouvelle, soit pour remplacer des emprunts indésirables aux langues étrangères $»^{2}$, publient les résultats de leurs travaux dans le Journal officiel. De plus, la loi Toubon introduite en 1994, recommande l'emploi de la langue française dans la vie sociale, où celle-ci garantit aux chercheurs et aux professionnels la possibilité « d'exprimer dans leurs langues les résultats de leurs travaux $»^{3}$. Dès 1996, les membres de la Commission d'enrichissement de la langue française collaborent avec des experts de dix-neuf domaines, dont le collège télécommunications ainsi que le collège culture et médias ${ }^{4}$, pour créer et promouvoir des équivalents français les plus exacts possible, afin d'éviter la nécessité d'employer des anglicismes. C'est cette même Commission qui est chargée de publier les unités créées dans le Journal officiel, celui-ci constituant une source de termes recommandés.

Par la présente recherche, nous nous proposons d'examiner dans quelle mesure ces indications officielles sont suivies par l'usage, ceci en confrontant la circulation en français des emprunts directs non intégrés (selon Esme Winter-Froemel, ce sont ceux qui présentent des structures non natives $)^{5}$ de ce champ lexical et des équivalents recommandés.

Pour ce faire, dans un premier temps, nous analyserons la présence des termes anglais du corpus dans des dictionnaires contemporains, afin d'observer l'adoption des recommandations officielles dans ces sources de référence. Dans un second temps, nous nous focaliserons sur la présence des anglicismes et leurs homologues autochtones dans le discours journalistique français (dans la presse non spécialisée), afin d'étudier à travers des cas concrets, la circulation des emprunts et d'examiner s'ils sont effectivement remplacés par les équivalents terminologiques de la langue d'accueil officiellement proposés.

\section{Le corpus}

Pour les besoins de cet article, nous nous appuyons sur un ensemble d'unités provenant essentiellement du répertoire de la rubrique Domaines et des fichiers Petit et grand écran ainsi que Etoiles et toiles de la série Vous pouvez le dire en français, publiés sur le site du Ministère de la Culture FranceTerme.fr ${ }^{6}$. Ces sources, qui résultent de la coopération

2 Les grandes lignes du dispositif d'enrichissement de la langue française in «Références 2018 : l'enrichissement de la langue Française » p. 3, disponible en ligne : https://www.culture.gouv.fr/Sites-thematiques /Langue-francaise-et-langues-de-France/Actualites/References-2018-1-enrichissement-de-la-langue -francaise, consulté le 17. 05. 2020.

Ibid.

4 Voir « Liste des membres des collèges (ou groupes d'experts) du dispositif d'enrichissement de la langue française en 2019 » disponible en pdf. sur un site du ministère de la Culture : http://www .culture.fr/Ressources/FranceTerme/Le-dispositif-d-enrichissement-de-la-langue-francaise, consulté le 17. 05. 2020.

5 Nous nous inspirons de l'étude approfondie de la classification des innovations introduites par le contact linguistique d'Esme Winter-Froemel (2009) « Les emprunts linguistiques : enjeux théoriques et perspectives nouvelles ", Neologica, 3, pp. 79-122, selon laquelle, en réalisant la stratégie de l'importation, les emprunts directs non intégrés, en opposition aux emprunts directs intégrés par « intégration immédiate » ou par « intégration plus tardive », présentent des structures non natives.

6 http://www.culture.fr/Ressources/FranceTerme/Domaines, consulté le 20. 03. 2020. 
des experts et des linguistes, visent à promouvoir le bon usage des termes français à la place des anglicismes intégrés dans la langue.

Vu que cet ensemble comporte des unités les plus communes et adoptées par la langue depuis longtemps, nous l'avons complété par des emprunts anglais liés à la production cinématographique, ceux-ci relevés dans le glossaire du blog spécialisé Apprendre le ciné$m a^{7}$ et dans les génériques de films contemporains tels que "Intouchables » (Olivier Nakache, Éric Toledano, France 2011), « Jusqu’à la garde » (Xavier Legrand, France 2017) ou « Mes provinciales» (Jean-Paul Civeyrac, France 2018).

Cette recherche de termes anglophones nous a permis d'en retenir soixante-douze dont cinquante-six comportent les équivalents publiés dans le Journal officiel - ce dernier groupe, englobant presque uniquement des expressions nominales, à l'exception d'un seul verbe ("transcribe ») constitue le corpus de notre analyse. L'annexe contenant une liste de mots complète et des résultats de recherche est jointe à la fin de l'article.

\section{Entre les recommandations et la terminologie : les dictionnaires}

En premier lieu, compte tenu des indications officielles de la Commission d'enrichissement de la langue française, nous avons vérifié la présence des termes équivalents qu'elle propose dans trois dictionnaires francophones en ligne : le Larousse ${ }^{8}$, le Dictionnaire de l'Académie Française ${ }^{9}$ et le Trésor de la Langue Française informatisé (TLFi) ${ }^{10}$. L'analyse des résultats que ces dictionnaires affichent, révèle qu'ils adoptent les recommandations officielles à des degrés variés. Ainsi, note-t-on que le Larousse propose les définitions de vingt-cinq anglicismes (dont le mot multiplex a un sens différent), parmi lesquelles : blockbuster, casting, flashback et rush contiennent une information sur la recommandation officielle d'employer un équivalent publié dans le Journal officiel. À son tour le Dictionnaire de l'Académie Française contient sept lexies et leurs définitions. Chacun de ces termes est accompagné d'une remarque sur la recommandation officielle, ce qui semble naturel, compte tenu de la coopération étroite entre l'Académie Française et la Commission d'enrichissement de la langue française. Le TLFi affiche quatorze définitions d'anglicismes (parmi lesquels : bonus track, casting, fade, multiplex, en un sens différent), dont quatre termes : perchman, playback, remake, rush, sont accompagnés d'une remarque sur l'équivalent recommandé dans le Journal officiel.

On aperçoit donc, que seulement quatre termes anglais apparaissent dans tous les dictionnaires mentionnés à la fois : coach, playback, remake et rush. Par ailleurs, deux termes : flashback et live, ne sont présents que dans le Dictionnaire de l'Académie Française et le Larousse, alors qu' uniquement le TLFi et le Larousse comportent les termes : dolly (camera), one man show, perchman, thriller et zoom. On constate ainsi que les dictionnaires pris

7 https://apprendre-le-cinema.fr/, consulté le 15. 03. 2020.

8 Le site web du dictionnaire : https://www.larousse.fr/dictionnaires/francais, mis au jour régulièrement.

9 Le site web du dictionnaire disponible en accès gratuit en ligne dès 2019 (9e éd.) : https://www.dictionnaire-academie.fr/.

10 Le site web du dictionnaire disponible en accès gratuit en ligne dès 2002 (éd. des années 1971-1994) : http ://atilf.atilf.fr/. 
en compte sont plutôt réticents à accepter les termes empruntés ; or, en les définissant, ils ne se réfèrent pas directement aux formes équivalentes officiellement recommandées.

\section{Entre les recommandations et l'usage : la presse}

Le deuxième pas de notre recherche consiste à vérifier dans la presse généraliste française, notamment dans les articles de Le Monde, Le Figaro, Aujourd'hui en France, L'Humanité et Libération, la présence des unités du corpus possédant leurs équivalents autochtones. Pour ce faire, nous avons eu recours à toutes les archives de la base journalistique Europresse, en retenant pour le besoin de notre recherche les attestations des unités en question dans la presse française, ce qui a permis de confronter la fréquence de leur emploi et celle de leurs équivalents autochtones, ainsi que d'indiquer la première apparition de l'emprunt. L'analyse qui comporte les utilisations des emprunts anglais et de leurs homologues français a démontré que quatre cas de figure sont possibles, à savoir :

- les unités qui n’apparaissent dans la presse que sous la forme empruntée ;

- les emprunts qui sont plus fréquents dans la presse que leurs équivalents français ;

- les termes autochtones qui dominent les anglicismes,

- les termes qui n'apparaissent dans la presse que sous la forme d'une dénomination française.

\subsection{Les termes qui n'apparaissent dans la presse que sous la forme empruntée}

Les termes de cette catégorie ne sont pas nombreux. En effet, même si des équivalents français sont recommandés par le Journal officiel, les emprunts sont utilisés dans la presse à la place des termes français. Dans notre corpus ce cas ne concerne que sept unités, dont le mot funsubbing, présenté ci-dessous :

(1)

«Célébrant des formes artistiques qui ont pour nom manga et anime (...), débattant de pratiques comme le scantrading ou le fansubbing ou sacrifiant au rite du cosplay, les visiteurs de cet hybride de festival culturel et de Salon commercial consacreront l'emprise de la culture populaire japonaise sur un nombre croissant de jeunes - et moins jeunes - Français » (Le Monde, 06. 07. 2006).

Ce terme apparaît dans la presse française dès 2006, faute de la dénomination consensuelle française. L'anglicisme maintient sa position dominante malgré l'apparition de son équivalent français qui semble résulter d'une création lexicale indépendante : sous-titrage sauvage, recommandé dès 2010. On peut supposer que fansubbing, qui est absent des dictionnaires susmentionnés et qui consiste en « l'établissement d'une version sous-titrée d'un film ou d'une série, réalisée sans autorisation par des amateurs (...) ${ }^{11}$, est une pratique, et par conséquent un terme, encore moderne et qui n'est pas suffisamment connu,

11 http://www.culture.fr/franceterme/terme/CULT543, consulté le 15. 06. 2020. 
d'où le recours à son équivalent étranger. De plus, malgré le fait que dans les deux langues les termes sont composés, l'unité anglaise est plus courte et ne constitue graphiquement qu'un seul mot, alors que son homologue français est plurisyllabique et il est graphiquement plus complexe. Par conséquent, la forme empruntée peut être préférée pour des raisons d'économie linguistique.

\subsection{Les emprunts qui sont plus fréquents dans la presse que leurs équivalents français}

Il est particulièrement fréquent que, dans la presse française, les emprunts anglais et leurs équivalents autochtones apparaissent parallèlement, mais en quantité différente. En effet, on note que les locuteurs français, aussi bien les cinéastes que les non professionnels, emploient volontiers les anglicismes soit pour faire preuve de leur professionnalisme, soit pour valoriser leur vouloir paraître. Il est donc possible d'en déduire que le recours aux termes anglais non seulement comble des lacunes lexicales, mais qu'il peut aussi résulter d'un choix dicté par des fins psychologiques.

Ce cas est bien illustré par l'exemple des mots home cinéma et cinéma à domicile. Le terme home cinéma, désignant un substitut du cinéma dans des conditions domestiques, s'est popularisé dans la presse française dès 1994. C'est un mot hybride, car il combine un élément anglais (home) avec un élément français (cinéma), qui a naturellement remplacé le mot anglais cinema grâce à l'adaptation phonétique. On note également que cet anglicisme était initialement employé entre guillemets ainsi que sous une forme inadaptée, ce qui a renforcé le concept de nouveauté, comme il est représenté dans un article publié dans Le Monde, le 14. 07. 1995 :

(2)

«La télévision à écran plat ne devrait prendre son véritable essor populaire qu'avec le développement du "home cinema"».

L'intégration de cet emprunt en français a suivi, si bien que celui-ci est apparu 1074 fois dans la presse française jusqu'au mois de juin 2020. En voici un exemple :

(3)

«Une qualité d'image exceptionnelle, mais des performances sonores médiocres. Pour y remédier, la solution s'est vite imposée : installer un système audio de type home cinéma, composé d'un ampli et de plusieurs haut-parleurs, ou s'équiper d'une barre de son, une enceinte toute en longueur à placer sous le téléviseur. » (Le Figaro, 09. 03. 2020).

En comparant la fréquence de la circulation de ce mot dans la presse avec le terme français recommandé, cinéma à domicile, qui y est apparu pour la première fois en 1964, on note qu'il n'a été employé que 143 fois. Constatons aussi que ce dernier n'est apparu que trois fois en 2020.

Un autre exemple de cette situation est constitué par les mots guest star (ou sa variante guest-star) et vedette invitée, parmi lesquels le premier est fréquemment employé dans la presse française, par exemple : 
(4)

«Sarkozy guest star des municipales» (Aujourd'hui en France, 09. 03. 2020).

On note que très souvent, comme dans l'exemple cité ci-dessus, ce terme figure dans des contextes qui dépassent celui de la production cinématographique, tels que la politique ou le sport. Le procédé de l'élargissement de sens de l'unité empruntée témoigne de l'assimilation sémantique du mot dans le vocabulaire cible. En même temps, on remarque que vedette invitée, son homologue français, qui dès 1982 est apparu dans la presse seulement 28 fois, et qui figure dans le Journal officiel depuis 2007, est employé uniquement dans le contexte du cinéma ainsi que de la télévision, par exemple :

(5)

" "Une vedette invitée spéciale" joue un rôle secondaire mais important dans Top of the Lake 2 : Nicole Kidman, dans un emploi de femme de son âge et le paraissant, qui quitte son mari pour une femme» (Le Monde, 15.08.2019).

De plus, on peut aussi trouver dans la presse une variante hybride de cet emprunt : star invitée, comme dans :

(6)

«Une élève s'ennuie durant le cours de mathématiques et imagine un avenir radieux où elle serait la reine de toutes les soirées, une star invitée partout » (Le Figaro, 27. 10. 2016).

Ce terme, qui résulte de l'adjonction de l'équivalent français mentionné ci-dessus à l'emprunt, admet un substantif anglo-américain star et un adjectif français invitée. Il est à noter que malgré la neutralité de genre des noms en anglais, l'adjectif garde ici sa forme féminine liée au substantif vedette.

Un exemple intéressant est aussi offert par les unités lexicales blockbuster et grosse machine, deux termes signifiant " production cinématographique à gros budget publicitaire, destinée à produire des profits record $»^{12}$, qui coexistent dans la presse française. Pourtant, les résultats affichés par Europresse révèlent une plus grande propension à la version anglaise, qui figure également dans le Larousse. En effet, blockbuster, est un terme très présent dans la presse française dès 1984, où il dépasse plus de deux fois le terme français officiellement recommandé grosse machine, présent dans le Journal officiel depuis 2006. D'ailleurs, c'est un mot bien intégré dans le système de la langue française, car il s'écrit sans guillemets et ne nécessite pas de glose explicative, comme on le constate dans l'extrait de Libération, ci-dessous :

(7)

«Avec la réouverture ce lundi des cinémas, distributeurs et exploitants vont tenter, malgré les contraintes sanitaires, de résorber leurs pertes tout en renouant avec le public. La pénurie de blockbusters pourrait favoriser les indépendants » (Libération, 22. 06. 2020).

De plus, l'extension du sens de cet anglicisme, qui dépasse aujourd'hui le domaine du cinéma, constitue aussi une preuve de son implantation au vocabulaire français. Comme

12 https://www.larousse.fr/dictionnaires/francais/blockbuster/10910221, consulté le 25. 06. 2020. 
exemple d'un tel emploi, voici le fragment d'un article sur une exposition des œuvres de Raphael Santi dans le Musée Condé du Domaine de Chantilly:

(8)

"L'exposition qui succède au blockbuster du Louvre est passionnante en ce qu'elle permet de confronter les deux titans, l'aîné et le cadet, et mieux vaut ne pas être trop nombreux dans les anciennes chambres du duc devenu le cabinet d'arts graphiques du musée » (Aujourd'hui en France, 08. 03. 2020).

D'autres exemples de ce type d'emploi fourmillent dans la presse française, ce qui permet de noter l'élargissement de sens du mot blockbuster qui est aujourd'hui utilisé pour désigner tous les phénomènes destinés à produire des profits témoignant d'un grand succès.

\subsection{Les termes autochtones qui dominent les anglicismes}

Dans ce groupe, le terme français non seulement apparaît plus fréquemment dans la presse française, mais il y est aussi attesté depuis plus longtemps. Il semble de plus que la préexistence ou la construction des termes autochtones peut contribuer à la préférence des dénominations françaises.

C'est le cas notamment des mots camera operator / camera person, et cadreur, dont les deux premiers n'apparaissent dans la presse française qu'une seule fois, en 1977 :

(9)

«Peu de femmes derrière les caméras. Pas assez, mais il y en a. On ne dit déjà plus, elles l'ont exigé, cameraman; ont dit camera person» (Le Monde, 30. 05. 1977).

Par contre, leur homologue français, cadreur, est apparu 553 fois dès 1954, notamment dans un article sur Quentin Tarantino :

(10)

«Extraits de films, interviews, séquences animées viennent souligner la singularité d’un réalisateur exigeant et enthousiaste, cadreur hors pair, dialoguiste nerveux, trop souvent réduit à son penchant pour la provocation» (Le Monde, 05. 06. 2020).

On peut donc supposer que la raison de la dominance d'une forme autochtone résulte du fait que l'équivalent français est un mot simple, dissyllabique, et qu'il est ainsi plus court que le mot anglais, celui-ci étant composé et plurisyllabique. De plus, la construction du terme français rend son sens plus explicite, car en constituant un dérivé du verbe cadrer par l'ajout du suffixe -eur qui indique un agent d'action, le mot exprime une essence de compétence du camera operator, qui consiste plus précisément à « maintenir le "cadre" choisi par le réalisateur au cours des multiples déplacements de la caméra ou des acteurs ${ }^{13}$.

Il est à remarquer cependant que c'est l'emprunt cameraman, le synonyme anglais de camera operator et camera person, qui concourt depuis longtemps avec l'équivalent

13 https://www.cnrtl.fr/definition/cadreur, consulté le 20. 06. 2020. 
français dans la presse, bien qu'il ne soit pas pris en compte dans le répertoire des termes publiés sur le site du Ministère de la Culture. En voici l'exemple de son usage :

(11)

«Les interventions de sa soeur, Tzeni Katsarou, et de ses enfants aujourd'hui quinquagénaires - le cameraman Nicolas Petsilas et la chanteuse Lenou - complètent ce portrait. » (Le Figaro, 03. 04. 2020).

Un autre exemple de cette catégorie est constitué par les mots biographical picture et son équivalent français film biographique, officiellement recommandé dans le Journal officiel dès 2008. On note que dès sa première apparition en 2004, le terme emprunté n'apparaît dans la base étudiée que 9 fois en tout. Ainsi, son homologue français présente 193 occurrences dès 1952, comme dans cet entretien avec Jonathan Pryce:

(12)

" J'ai d'abord voulu refuser le rôle. Je n'étais pas enthousiaste à l'idée de faire un film biographique classique. » (Le Figaro, 20. 12. 2019).

La structure analogique et similaire (un adjectif suivi d'un nom) de ces deux mots et l'antériorité de l'emploi du film biographique dans la presse par rapport à l'apparition de l'emprunt, peuvent contribuer à la préférence accordée au terme français.

\subsection{Les termes qui n'apparaissent dans la presse que sous la forme d'une dénomination française}

Comme il a déjà été dit, le caractère international de la cinématographie favorise l'emploi des termes anglais - par conséquent la presse aussi y fait recours de préférence. Ainsi, les emplois des équivalents français sont rares : dans notre corpus ce cas ne concerne que sept unités, dont deux sont ci-dessous présentées.

Le premier exemple concerne le couple plan rapproché et medium close-up, désignant le cadre qui, afin de mettre l'accent sur les personnages, les taille au niveau de la ceinture. La recherche de ces deux unités dans la presse révèle qu'un anglicisme est évincé par son homologue français, qui figure dans le Journal officiel dès 2000. En voici un exemple:

(13)

" Nous sommes autorisés à photographier, mais pas en plan rapproché, "par respect pour les familles” qui parfois se manifestent, explique Irina Bogolepova» (Le Figaro, 21. 02. 2020).

On remarque que le terme français, appelé aussi plan américain, ${ }^{14}$ n'est pas moins précis que medium close-up et s'inscrit dans une large gamme des dénominations françaises des plans appliquées depuis longtemps dans les arts visuels. Ainsi, il s'intègre naturellement à ce répertoire, ce qui peut s'expliquer par la réticence à employer le mot étranger.

Un autre exemple illustrant cette situation est constitué par les mots câbliste et cableperson, dénommant un professionnel spécialisé dans la manipulation des câbles d'une

14 https://www.cnrtl.fr/definition/plan, (consulté le 20. 06. 2020). 
caméra lors d'une prise de vues. On note que câbliste, un terme d'une insignifiante circulation dans la presse généraliste, a cependant éliminé l'anglicisme. On en voit un exemple dans l'extrait ci-dessous.

\section{(14)}

«Le plus extraordinaire, dans ses souvenirs très précis, c’était son entrée en cinéma, ce passage du statut d'électricien (de câbliste, plutôt) à celui de metteur en scène, une fonction qui ne valait pas mieux que celle de contremaître " (Libération, 27. 08. 2003).

Soulignons que les deux termes présentent une construction analogue : ils comportent un nom d'instrument - en anglais : cable et en français : câble, et une " marque " de son opérateur - en anglais il s'agit du substantif person et en français d'un suffixe nominal d'agent -iste. Ainsi, il est probable que c'est l'économie linguistique qui contribue à la fréquence de l'usage de l'unité française, car câbliste est un mot simple et dissyllabique, alors que le terme emprunté anglais est un mot composé et tétrasyllabique. Il convient de noter que la réticence aux mots anglais se manifeste très souvent lorsque le terme français résulte du procédé de la néologie de sens, qui permet au signifiant préexistant de « s'adapter » à la réalité nouvelle et de désigner un signifiant nouveau.

\section{Conclusions}

L’objectif de notre article était d'examiner, sur l'exemple du domaine de la cinématographie, l'application pratique des indications résultant de la politique officielle visant l'usage en français des termes équivalents francophones au lieu des termes anglo-américains. Nous avons d'abord étudié la présence des emprunts du corpus dans trois dictionnaires contemporains pour évaluer ensuite les préférences de leur utilisation dans le discours journalistique.

Notre recherche a permis de confirmer que les travaux des institutions chargées de la création et de la promotion des termes autochtones encouragent la réduction des anglicismes dans le domaine du cinéma, ainsi que l'emploi des équivalents français. Néanmoins, les anglicismes apparaissent dans les dictionnaires qui adoptent les recommandations officielles à des degrés variés et dominent aussi dans les sources françaises conçues pour les destinateurs internationaux, notamment dans les génériques de films ; ceux-ci sont également employés par les médias. On observe par ailleurs que les termes qui ne possèdent pas leurs équivalents autochtones présentent quand même une circulation notable dans la presse, par exemple final cut ${ }^{15}$, flash-forward ${ }^{16}$ ou remake ${ }^{17}$. Qui plus est, ces mots semblent

15 Le mot qui apparaît 365 fois dans la presse française (consulté sur Europresse), par exemple : « Finalement, j' ai obtenu le droit du final cut, ce qui est très rare pour un premier film en Chine. " (Le Monde, 26. 12. 2019).

16 Le mot qui apparaît 59 fois dans la presse française (consulté sur Europresse), par exemple : «Certains cinéastes ont inventé des notions pour penser cela, tel Edouard de Laurot, dont j'aime beaucoup la notion de prolepse, qui correspond à la figure cinématographique du flash-forward, et qui consiste à déceler dans le présent ce qui pourrait favoriser un futur plus juste. » (Libération, 18. 01. 2020).

17 Le mot qui apparaît dans 10135 occurrences dans la presse française (consulté sur Europresse), entre autres dans L'Humanité : "Triste remake de “Touche pas à mon pote", le gentil ami blanc qui défend on ami "non blanc". » (L'Humanité, 15. 06. 2020). 
être bien intégrés dans le système de la langue française : la preuve en est qu'ils n'apparaissent pas entre les guillemets ni ne sont accompagnés d'une glose explicative.

L'analyse de la circulation des mots dans la presse montre que le plus souvent les équivalents français coexistent avec les termes empruntés eux-mêmes bien intégrés dans la langue française, vu qu'ils sont rarement accompagnés de gloses explicatives ou de guillemets. Dans le cadre de notre corpus cela concerne trente-huit unités, dont vingt concernent le cas de l'emploi plus fréquent du mots anglais et dix-huit témoignent de la préférence pour l'équivalent français. Il est bien évident que « lorsqu'il y a coexistence et concurrence d'emploi, il n'y a pas forcément synonymie » (Petiot 1987 : 302) ; ainsi, note-t-on que les raisons de la concurrence entre les termes français et les anglicismes s'expliquent par des valeurs stylistiques résultant de l'emploi du mot étranger et par la tendance à l'économie linguistique. Comme le remarque Valérie Saugera, le fait de remplacer les emprunts par leurs équivalents français n'est pas uniquement une question de procédé linguistique, mais aussi d'une opération entre la sémantique et la pragmatique, car il n' existe jamais de véritables synonymes, en revanche il est toujours question de valeurs extra-stylistiques qui interviennent (Saugera, 2017). Très souvent il est impossible de remplacer un anglicisme par un terme français sans l'ingérence du sens et des connotations. Comme le note Jean-Marc Chadelat, les emprunts ne jouent pas seulement le rôle de combler des lacunes verbales, mais ils véhiculent aussi des valeurs stylistiques (Chadelat 1966 : 17). Ainsi, l'écart entre l'usage des mots et les recommandations officielles peut résulter d'un choix délibéré, fait par des journalistes à la recherche d'une expressivité particulière. Il n'est pas non plus exclu que, surtout dans le cas des mots courts, moins techniques et faciles à prononcer, comme cast, guest star, trailer etc., l'emprunt découle de la tendance à l'économie linguistique.

On note cependant, que le contournement dans l'usage journalistique des équivalents autochtones n'est pas fréquent, et qu'il a lieu dans le cas de sept termes nouveaux et inconnus pour les locuteurs non professionnels. L'éviction de l'emprunt par la lexie française est également très rare : dans le corpus elle ne concerne que sept unités. Elle résulte principalement de l'économie linguistique, lorsqu'un équivalent français grâce à sa structure explique mieux ou précise davantage le sens du mot étranger, ou quand sa présence dans la presse précède considérablement l'apparition de l'emprunt. De plus, il est à noter que les homologues français sont plus souvent considérés comme inséparablement associés au domaine du cinéma que les emprunts anglais, car ce sont ces derniers qui figurent plus fréquemment hors du contexte de la cinématographie. Cet élargissement du sens dans le cas des termes anglais fait preuve de leur implantation dans la langue française.

Ce travail visant à vérifier l'application des recommandations officielles met en lumière le fait que, malgré l'engagement des institutions dont le but est de défendre la langue française et de promouvoir les termes autochtones, il y a des emprunts qui n'ont pas toujours leurs équivalents en français, ou qui, malgré les démarches entreprises, dominent, voire évincent, les homologues autochtones. Cet article ne vise qu'à signaler un besoin d'une recherche plus vaste visant la confrontation des emprunts et des lexies officiellement recommandées dans le contexte de domaines qui se caractérisent par un contact intense entre les langues. Une telle étude demanderait des analyses approfondies tant sur la structure de ces termes que sur leurs valeurs stylistiques et pragmatiques afin 
de prendre en considération les "stratégies fondamentales ${ }^{18}$ des créations lexicales résultant de l'innovation analogique ou indépendante dans un domaine précis.

\title{
BIBLIOGRAPHIE
}

Chadelat, J.-M. (1996) : « Pour une sociolinguistique de l'emprunt lexical : l'exemple des emprunts français en anglais ». Cahiers de l'APLIUT, vol. 15, numéro 4, 1996. pp. 16-27. <https://doi.org/10.3406/apliu.1996.985>, consulté le 24. 04. 2020.

Giraud, J. (1967) : «Terminologie du cinéma » Meta 12 (4) pp. 118-124, <https://id.erudit.org/iderudit /002919ar>, consulté le 30. 04. 2020.

Pergnier, M. (1989) : Les anglicismes. Paris : Presses Universitaires de France.

Petiot, G. (1987) : «Le cinéma américain et la langue française », Meta 32 (3) pp. 299-305, https://doi .org/10.7202/003829ar.

Rapport annuel 2018 de la Commission d'enrichissement de la langue française

Saugera, V. (2017) : « La fabrique des anglicismes », Travaux de linguistique 2 (75), pp.59-79, <https://www .cairn.info/revue-travaux-de-linguistique-2017-2-page-59.html.>, consulté le 02. 03. 2020.

Winter-Froemel, E. (2009) : «Les emprunts linguistiques : enjeux théoriques et perspectives nouvelles », Neologica, 3, p. 79-122.

http://www.academie-francaise.fr

http://www.atilf.fr/tlfi .

http://www.culture.fr/franceterme

http://www.europresse.com

http://www.granddictionnaire.com.

http://www.linfodrome.com

https://apprendre-le-cinema.fr

https://www.cnrtl.fr.

https://www.culture.gouv.fr

https://www.francetvinfo.fr

https://www.larousse.fr.

https://www.legifrance.gouv.fr

\author{
Weronika Woźniak \\ Université de Łódź \\ Institut d'Études Romanes \\ weronika.wozniak@edu.uni.lodz.pl
}

18 Pour voir plus : Winter-Froemel, E. (2009) : « Les emprunts linguistiques : enjeux théoriques et perspectives nouvelles », Neologica, 3, pp. 79-122. 
L'annexe

\begin{tabular}{|c|c|c|c|c|c|c|c|c|c|c|c|c|c|c|c|}
\hline 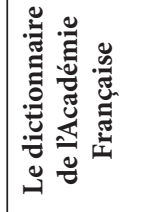 & 1 & 1 & 1 & 1 & 1 & 1 & 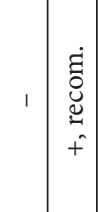 & 1 & 1 & 1 & 1 & $1+$ & 1 & 1 & + \\
\hline 1: & + & 1 & 1 & 1 & 1 & 1 & $\begin{array}{l}\tilde{\Xi} \\
\stackrel{0}{\sharp} \\
+ \\
+\end{array}$ & + & + & + & 1 & 1 & 1 & 1 & $\begin{array}{l}\dot{\Xi} \\
\stackrel{0}{0} \\
+ \\
+\end{array}$ \\
\hline 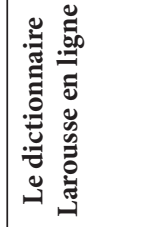 & 1 & + & 1 & 1 & 1 & 1 & ++ & + & ++ & 1 & 1 & $1+$ & 1 & 1 & 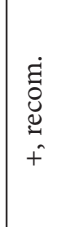 \\
\hline 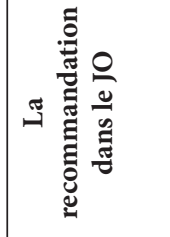 & 1 & 1 & 1 & 1 & 1 & 1 & 1 & 1 & 1 & 1 & 1 & 1 & \begin{tabular}{l|l}
$\vec{\Xi}$ & \\
$\stackrel{N}{0}$ & \\
0 &
\end{tabular} & 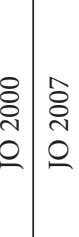 & $\begin{array}{l}8 \\
\stackrel{0}{0} \\
0 \\
0\end{array}$ \\
\hline 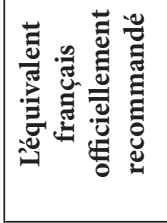 & 1 & 1 & 1 & 1 & 1 & \begin{tabular}{l|l}
1 & 1
\end{tabular} & 1 & 1 & 1 & 1 & 1 & 1 & 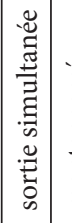 & 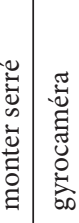 & 艺 \\
\hline 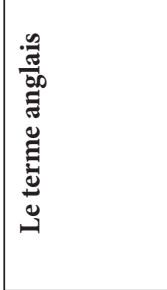 & 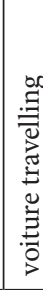 & 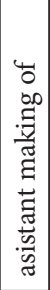 & 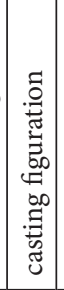 & 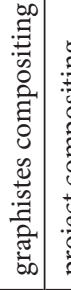 & 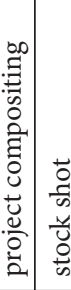 & 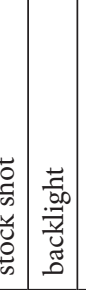 & 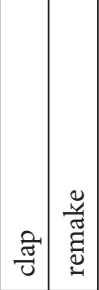 & $\mid \begin{array}{l}\vec{\Xi} \\
\overline{\vec{Z}} \\
\Xi\end{array}$ & 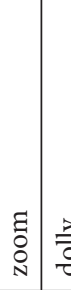 & 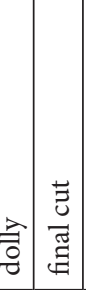 & 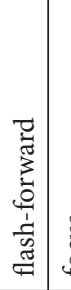 & $\mid$ & 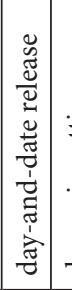 & 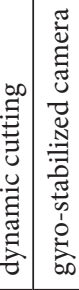 & $\begin{array}{l}\overline{5} \\
\bar{z} \\
\end{array}$ \\
\hline 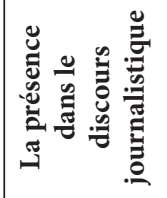 & \multicolumn{12}{|c|}{ 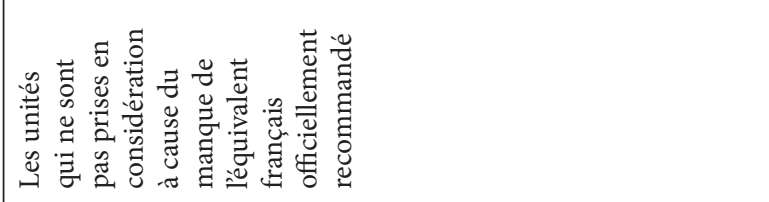 } & \multicolumn{3}{|c|}{ 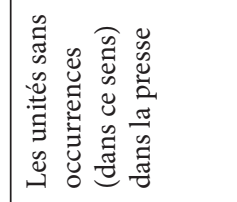 } \\
\hline
\end{tabular}




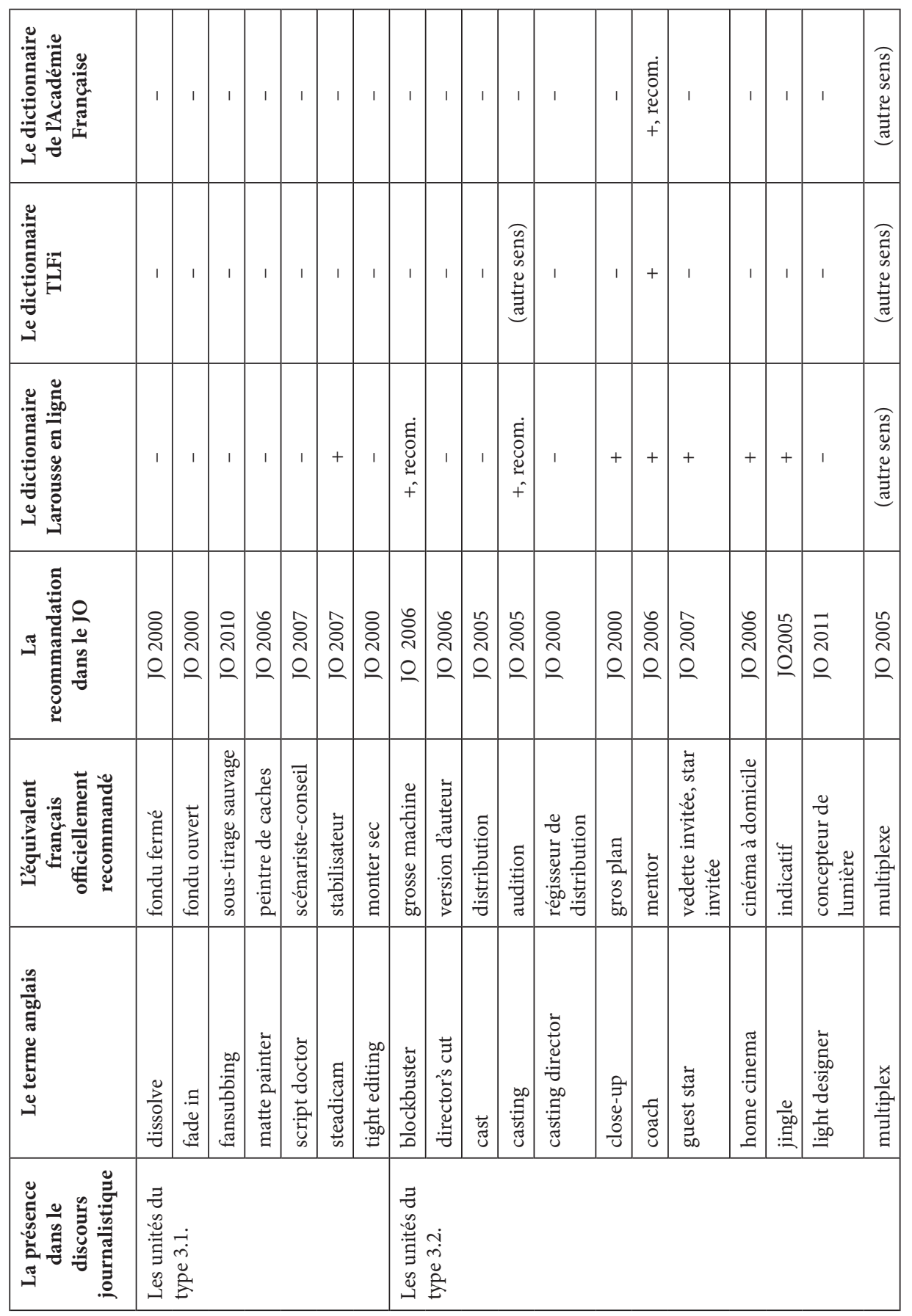




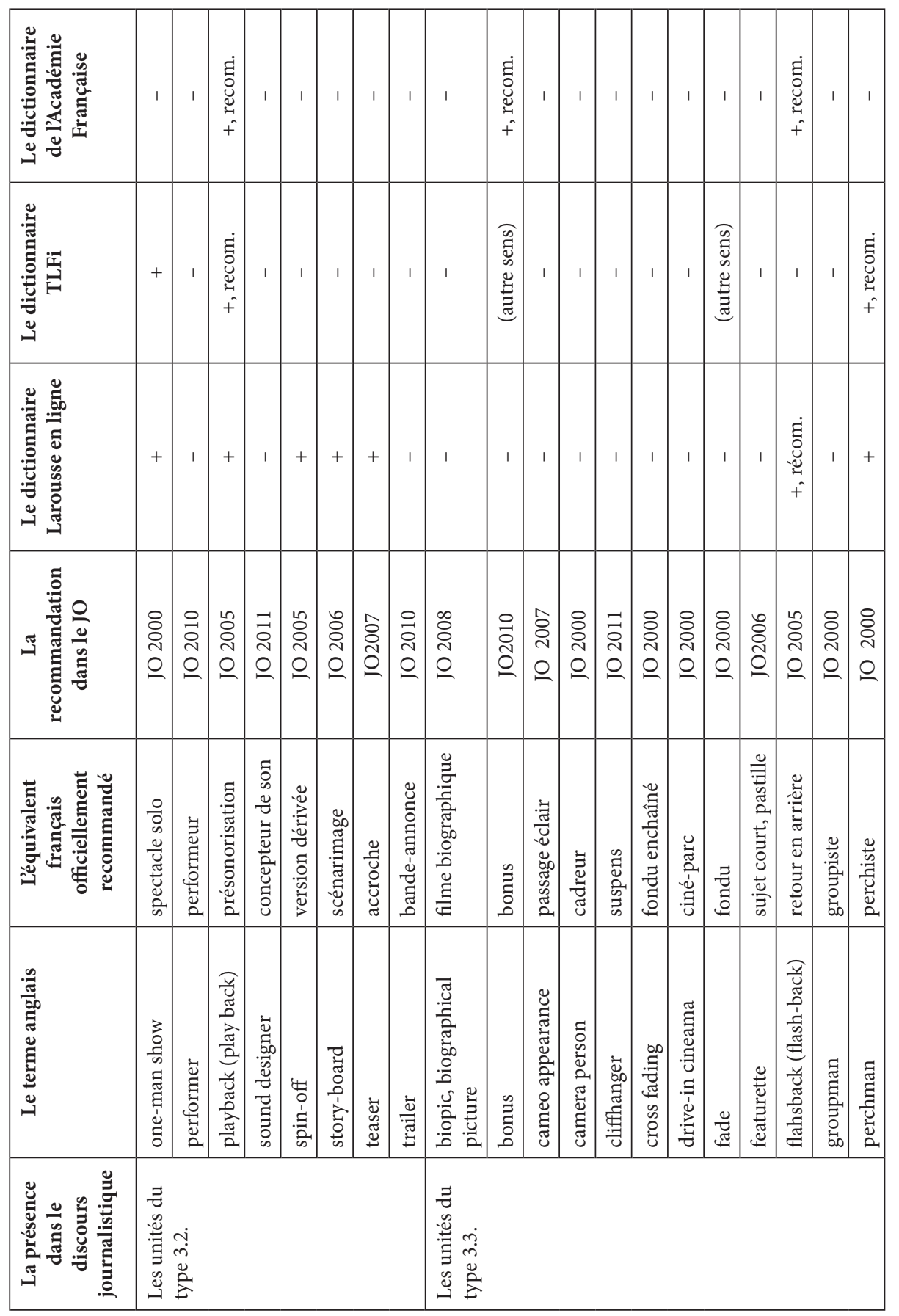




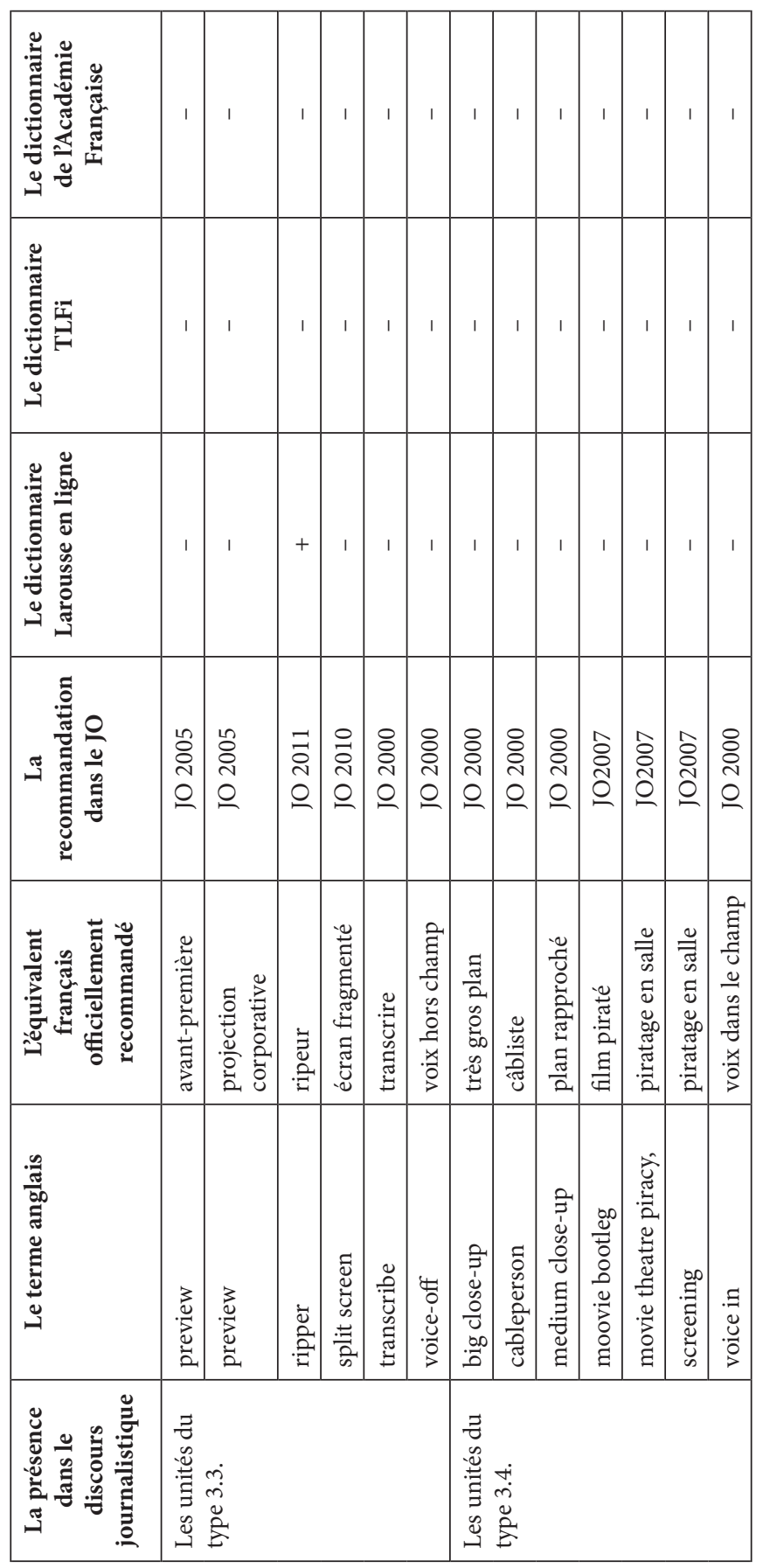

